\title{
Statistical limit superior and limit inferior in intuitionistic fuzzy normed spaces
}

\author{
Mohammad Ali Alghamdi ${ }^{1}$, Abdullah Alotaibi ${ }^{1}$, Qutubuddin Mohammad Danish Lohani ${ }^{2}$ and \\ Mohammad Mursaleen ${ }^{3^{*}}$
}

\author{
* Correspondence: \\ mursaleenm@gmail.com \\ ${ }^{3}$ Department of Mathematics, \\ Aligarh Muslim University, Aligarh \\ 202002, India \\ Full list of author information is \\ available at the end of the article
}

\begin{abstract}
Recently, the concepts of statistical convergence, ideal convergence and lacunary statistical convergence have been studied in intuitionistic fuzzy normed spaces. In this article, we study the concepts of statistical limit superior and statistical limit inferior in intuitionistic fuzzy normed spaces. We also give an example to compute these points in intuitionistic fuzzy normed spaces.

AMS Subject Classification (2000): 40A05; 40D25; 11B05; 60H10; 60B99; $26 \mathrm{AO} 03$.

Keywords: $t$-norm, $t$-conorm, fuzzy numbers, intuitionistic fuzzy normed space, statistical convergence, statistical boundedness, statistical limit point, statistical cluster point, statistical limit superior, statistical limit inferior.
\end{abstract}

\section{Introduction and preliminaries}

The concept of statistical convergence was first introduced by Fast [1] which was extended for double sequences in $[2,3]$. In particular, active researches on this topic were started after the study of Fridy [4]. Many of the results of the theory of ordinary convergence have been extended to the theory of statistical convergence by using the notion of density. For instance, Fridy [5] introduced the concept of statistical limit points and Fridy and Orhan [6] introduced the statistical analogs of limit superior and limit inferior of a sequence of real numbers. Recently, statistical convergence and some of its related concepts for fuzzy numbers have been studied in [7-9]. Quite recently, the idea of statistical convergence in intuitionistic fuzzy normed spaces for single sequences has been studied in [10,11]; and for double sequences by Mursaleen and Mohiuddine [12,13].

Recently, Saadati and Park [14] introduced the notion of intuitionistic fuzzy normed space and quite recently, in $[15,16]$ the concepts of intuitionistic fuzzy 2-normed and intuitionistic fuzzy 2-metric spaces have been introduced and studied. Certainly there are some situations where the ordinary norm does not work and the concept of intuitionistic fuzzy norm seems to be more suitable in such cases.

In this article, we study the concept of statistical limit superior and statistical limit inferior in intuitionistic fuzzy normed spaces. An example is demonstrated to determine these points in intuitionistic fuzzy normed space. We observe that our results are analogous to the results of Fridy and Orhan [6] but proofs are somewhat different when we deal with these concepts in intuitionistic fuzzy normed spaces.

(c) 2012 Alghamdi et al; licensee Springer. This is an Open Access article distributed under the terms of the Creative Commons Attribution License (http://creativecommons.org/licenses/by/2.0), which permits unrestricted use, distribution, and reproduction in any medium, provided the original work is properly cited. 
We recall some basic definitions and notations.

Definition $1.1[14]$. A binary operation *: $[0,1] \times[0,1] \rightarrow[0,1]$ is said to be a continuous t-norm if it satisfies the following conditions:

(a) * is associative and commutative,

(b) * is continuous,

(c) $a^{*} 1=a$ for all $a \in[0,1]$,

(d) $a^{*} b \leq c * d$ whenever $a \leq c$ and $b \leq d$ for each $a, b, c, d \in[0,1]$.

For example, $a^{*} b=\max \{a+b-1,0\}, a^{*} b=a b$ and $a^{*} b=\min \{a, b\}$ on $[0,1]$ are $t$-norms.

A binary operation $\diamond:[0,1] \times[0,1] \rightarrow[0,1]$ is said to be a continuous $t$-conorm if it satisfies the conditions (a), (b), (d) as above and $a \diamond 0=a$ for all $a \in[0,1]$.

For example, $a \diamond b=\min \{a+b, 1\}$ and $a \diamond b=\max \{a, b\}$ on [0,1] are $t$-conorms.

Definition 1.2 [14]. The five-tuple $(X, \mu, v, *, \diamond)$ is said to be an intuitionistic fuzzy normed space (for short, IFNS) if $X$ is a vector space," is a continuous $t$-norm, $\diamond$ is a continuous $t$-conorm, and $\mu, v$ are fuzzy sets on $X \times(0, \infty)$ satisfying the following conditions. For every $x, y \in X$ and $s, t>0$,
(a) $\mu(x, t)+v(x, t) \leq 1$,
(b) $\mu(x, t)>0$,
(c) $\mu(x, t)=1$ if and only if $x=0$,
(d) $\mu(\alpha x, t)=\mu\left(x, \frac{t}{|\alpha|}\right)$ for each $\alpha \neq 0$,
(e) $\mu(x, t) * \mu(y, s) \leq \mu(x+y, t+s)$,
(f) $\mu(x, \cdot):(0, \infty) \rightarrow[0,1]$ is continuous,
(g) $\lim _{t \rightarrow \infty} \mu(x, t)=1$ and $\lim _{t \rightarrow 0} \mu(x, t)=0$,
(h) $v(x, t)<1$,
(i) $v(x, t)=0$ if and only if $x=0$,
(j) $v(\alpha x, t)=v\left(x, \frac{t}{|\alpha|}\right)$ for each $\alpha \neq 0$,
(k) $v(x, t) \diamond v(y, s) \geq v(x+y, t+s)$,
(l) $v(x, \cdot):(0, \infty) \rightarrow[0,1]$ is continuous,
(m) $\lim _{t \rightarrow \infty} v(x, t)=0$ and $\lim _{t \rightarrow 0} v(x, t)=1$.

In this case $(\mu, v)$ is called an intuitionistic fuzzy norm.

Example. Suppose that $(X,\|\|)$ is a normed space and let $a^{*} b=a b$ and $a \diamond b=\min$ $\{a+b, 1\}$ for all $a, b \in[0,1]$. For all $x \in X$ and every $t>0$, consider

$$
\mu(x ; t):=\frac{t}{t+\|x\|} \quad \text { and } \quad v(x ; t):=\frac{\|x\|}{t+\|x\|} .
$$

Then $(X, \mu, v, *, \diamond)$ is an intuitionistic fuzzy normed space.

Definition 1.3 [14]. Let $(X, \mu, v, *, \diamond)$ be an intuitionistic fuzzy normed space. Then a sequence $x=\left(x_{n}\right)$ is said to be convergent to $L \in X$ with respect to the intuitionistic fuzzy norm $(\mu, v)$ if for every $\epsilon>0$ and $t>0$, there exists a positive integer $k_{\mathrm{o}}$ such that 
$\mu\left(x_{n}-L ; t\right)>1-\epsilon$ and $v\left(x_{n}-L ; t\right)<\epsilon$, whenever $n \geq k_{\mathrm{o}}$. In this case, we write $(\mu, v)$-lim $x=L$ or $\underset{x_{n}}{\stackrel{(\mu, v)}{\rightarrow}} L$ as $n \rightarrow \infty$.

Definition 1.4 [14]. Let $\left(X, \mu, v,{ }^{*}, \diamond\right)$ be an intuitionistic fuzzy normed space. Then a sequence $x=\left(x_{n}\right)$ is said to be a Cauchy sequence with respect to the intuitionistic fuzzy norm $(\mu, v)$ if for every $\epsilon>0$ and $t>0$, there exists a positive integer $k_{\mathrm{o}}$ such that $\mu\left(x_{n}-x_{m} ; t\right)>1-\epsilon$ and $v\left(x_{n}-x_{m} ; t\right)<\epsilon$ for all $n, m \geq k_{\mathrm{o}}$.

Definition 1.5 [17]. If $K$ is a subset of $\mathbb{N}$, then the natural density of $K$ denoted by $\delta(K)$, is defined as

$$
\delta(K):=\lim _{n} \frac{1}{n}|\{k \leq n: k \in K\}|,
$$

where the vertical bars denote the cardinality of the enclosed set.

Definition 1.6 $[4,18]$. A sequence $x=\left(x_{n}\right)$ of numbers is said to be statistically convergent to $L$ if

$$
\delta\left(\left\{k \leq n:\left|x_{k}-L\right| \geq \epsilon\right\}\right)=0
$$

for every $\epsilon>0$. In this case we write $s t$ - $\lim x=L$.

Definition $1.7[5,6]$. A sequence $x=\left(x_{n}\right)$ of numbers is said to be statistically bounded if there is a number $B$ such that

$$
\delta\left(\left\{k \leq n:\left|x_{k}\right| \geq B\right\}\right)=0 .
$$

Definition 1.8 [5]. If $\left\{x_{k(j)}\right\}$ is a subsequence of $x=\left(x_{k}\right)$ and $K:=\{k(j): j \in \mathbb{N}\}$, then we abbreviate $\left\{x_{k(j)}\right\}$ by $\{x\}_{K}$. If $\delta(K)=0$ then $\{x\}_{K}$ is called a subsequence of density zero or a thin subsequence. On the other hand, $\{x\}_{K}$ is a nonthin subsequence of $x$ if $K$ does not have density zero.

Definition 1.9 [10,12]. Let $\left(X, \mu, v,{ }^{*}, \diamond\right)$ be an intuitionistic fuzzy normed space. We say that a sequence $x=\left(x_{k}\right)$ is statistically convergent to $L \in X$ with respect to the intuitionistic fuzzy normed $(\mu, v)$ provided that for every $t>0$ and $b \in(0,1)$

$$
\delta\left(\left\{k \leq n: \mu\left(x_{k}-L ; t\right)<1-b \text { or } v\left(x_{k}-L ; t\right)>b\right\}\right)=0 .
$$

In this case we write $s t_{(\mu, v)}-\lim x=L$.

\section{Statistical limit superior and inferior in IFNS}

In this section, we define limit point, statistical limit point, statistical cluster point, statistical limit superior, and statistical limit inferior in intuitionistic fuzzy normed spaces and demonstrate through an example how to compute these points in a IFN-spaces.

Definition 2.1. A sequence $x$ in an intuitionistic fuzzy normed space $(X, \mu, v, *, \diamond)$ is said to be statistically bounded if there exists some $t_{\mathrm{o}}>0$ and $b \in(0,1)$ such that $\delta(\{k$ : $\mu\left(x_{k} ; t_{\mathrm{o}}\right)>1-b$ or $\left.\left.v\left(x_{k} ; t_{\mathrm{o}}\right)<b\right\}\right)=0$.

Definition 2.2. Let $(X, \mu, v, *, \oslash)$ be an intuitionistic fuzzy normed space. Then $l \in X$ is called a limit point of the sequence $x=\left(x_{k}\right)$ with respect to the intuitionistic fuzzy norm $(\mu, v)$ provided that there is a subsequence of $x$ that converges to $l$ with respect to the intuitionistic fuzzy norm $(\mu, v)$. Let $L_{(\mu, v)}(x)$ denotes the set of all limit points of the sequence $x$ with respect to the intuitionistic fuzzy norm $(\mu, v)$.

Definition 2.3. Let $(X, \mu, v, *, \diamond)$ be an intuitionistic fuzzy normed space. Then $\xi \in X$ is called a statistical limit point of the sequence $x=\left(x_{k}\right)$ with respect to the 
intuitionistic fuzzy norm $(\mu, v)$ provided that there is a nonthin subsequence of $x$ that converges to $\xi$ with respect to the intuitionistic fuzzy norm $(\mu, v)$. In this case we say $\xi$ is a $s t_{(\mu, v)}$-limit point of sequence $x=\left(x_{k}\right)$. Let $\Lambda_{(\mu, v)}(x)$ denotes the set of all $s t_{(\mu, v)^{-}}$ limit points of the sequence $x$.

Definition 2.4. Let $(X, \mu, v, *, \diamond)$ be an intuitionistic fuzzy normed space. Then $\eta \in X$ is called a statistical cluster point of the sequence $x=\left(x_{k}\right)$ with respect to the intuitionistic fuzzy norm $(\mu, v)$ provided that for every $t_{\mathrm{o}}>0$ and $a \in(0,1)$,

$$
\bar{\delta}\left(\left\{k \leq n: \mu\left(x_{k}-\eta ; t_{0}\right)>1-a \text { or } v\left(x_{k}-\eta ; t_{0}\right)<a\right\}\right)=0 .
$$

In this case we say $\eta$ is a $s t_{(\mu, v)}$-cluster point of the sequence $x$. Let $\Gamma_{(\mu, v)}(x)$ denotes the set of all $s t_{(\mu, v)}$-cluster points of the sequence $x$.

Definition 2.5. For a sequence $x$ in an intuitionistic fuzzy normed space $(X, \mu, v, *, \nabla)$, we define the sets $B_{x}^{(\mu, \nu)}$ and $A_{x}^{(\mu, \nu)}$ by

$$
\begin{aligned}
& B_{x}^{(\mu, \nu)}:=\left\{b \in(0,1): \delta\left(\left\{k: \mu\left(x_{k} ; t\right)<1-b \text { or } v\left(x_{k} ; t\right)>b\right\}\right) \neq 0\right\} \\
& A_{x}^{(\mu, \nu)}:=\left\{a \in(0,1): \delta\left(\left\{k: \mu\left(x_{k} ; t\right)>1-a \text { or } v\left(x_{k} ; t\right)<a\right\}\right) \neq 0\right\}
\end{aligned}
$$

If $x$ is a real number sequence then the statistical limit superior of $x$ with respect to the intuitionistic fuzzy norm $(\mu, v)$ is defined by

$$
s t_{(\mu, \nu)}-\lim \sup x:= \begin{cases}\sup B_{x}^{(\mu, \nu)} & \text { if } B_{x}^{(\mu, \nu)} \neq \emptyset, \\ 0 & \text { if } \quad B_{x}^{(\mu, \nu)} \neq \emptyset .\end{cases}
$$

And the statistical limit inferior of $x$ with respect to the intuitionistic fuzzy norm $(\mu, v)$ is defined by

$$
s t_{(\mu, \nu)}-\liminf x:=\left\{\begin{array}{rrrr}
\inf A_{x}^{(\mu, \nu)} \text { if } A_{x}^{(\mu, \nu)} & \neq \varnothing, \\
1 & \text { if } & A_{x}^{(\mu, \nu)} & \neq \varnothing .
\end{array}\right.
$$

Example. A simple example will help to illustrate the concepts just defined. Let the sequence $x=\left(x_{k}\right)$ be defined by

$$
x_{k}:= \begin{cases}2 k, & \text { if } k \text { is an odd square, } \\ -1, & \text { if } k \text { is an even square, } \\ 1 / 2, & \text { if } k \text { is an odd nonsquare, } \\ 0, & \text { if } k \text { is an even nonsquare. }\end{cases}
$$

Let $\mu\left(x_{k} ; t\right)=\frac{t}{t+\left|x_{k}\right|}$ and $v\left(x_{k} ; t\right)=\frac{\left|x_{k}\right|}{t+\left|x_{k}\right|}$.

The above sequence is clearly unbounded with respect to $(\mu, v)$. On the other hand, it is statistically bounded with respect to $(\mu, v)$. For this,

$$
\begin{aligned}
& \delta\left(\left\{k \leq n: \mu\left(x_{k} ; t_{o}\right)<1-b \text { or } v\left(x_{k} ; t_{o}\right)>b\right\}\right) \\
= & \delta\left(\left\{k \leq n: \frac{t_{o}}{t_{o}+\left|x_{k}\right|}<1-b \text { or } \frac{\left|x_{k}\right|}{t_{o}+\left|x_{k}\right|}>b\right\}\right), \\
= & \delta\left(\left\{k \leq n:\left|x_{k}\right|>\frac{b t_{o}}{1-b}\right\}\right) .
\end{aligned}
$$


Since $0<b<1, \frac{1}{b}-1>0$. Choose $t_{\mathrm{o}}=\frac{1-b}{3 b}$. Then $t_{\mathrm{o}}>0$ and

$$
\begin{aligned}
\delta\left(\left\{k \leq n: \mu\left(x_{k} ; t_{0}\right)<\right.\right. & \left.\left.1-b \text { or } v\left(x_{k} ; t_{0}\right)>b\right\}\right)=\delta\left(\left\{k \leq n:\left|x_{k}\right|>\frac{b}{1-b} \times \frac{1-b}{3 b}=\frac{1}{3}\right\}\right) \\
& =\delta\left(\left\{k \leq n:\left|x_{k}\right|>\frac{1}{3}\right\}\right)=\lim _{n \rightarrow \infty} \frac{1}{n} \times \sqrt{n}=0
\end{aligned}
$$

Hence it is statistically bounded with respect to $(\mu, v)$.

To find $B_{x}^{(\mu, v)}$, we have to find those $b \in(0,1)$ such that

$$
\delta\left(\left\{k \leq n: \mu\left(x_{k} ; t\right)<1-b \text { or } v\left(x_{k} ; t\right)>b\right\}\right) \neq 0 .
$$

Now,

$$
\begin{aligned}
& \delta\left(\left\{k \leq n: \mu\left(x_{k} ; t\right)<1-b \text { or } v\left(x_{k} ; t\right)>b\right\}\right) \\
=\delta(\{k & \left.\left.\leq n: \frac{t}{t+\left|x_{k}\right|}<1-b \text { or } \frac{\left|x_{k}\right|}{t+\left|x_{k}\right|}>b\right\}\right), \\
= & \delta\left(\left\{k \leq n:\left|x_{k}\right|>\frac{b t}{1-b}\right\}\right) .
\end{aligned}
$$

We can easily choose any $t>0$ as $t<\frac{1}{3}\left(\frac{1}{b}-1\right)$ for $0<b<1$, so that

$$
0<\frac{b t}{1-b}<\frac{b}{1-b} \times \frac{1-b}{3 b}=\frac{1}{3} \text {. }
$$

Therefore

$$
\delta\left(\left\{k \leq n:\left|x_{k}\right|>\frac{b t}{1-b}\right\}\right)=\delta\left(\left\{k \leq n:\left|x_{k}\right|>r=\frac{b t}{1-b}\right\}\right),
$$

and by the above condition $r \in(0,1)$. Now the number of members of the sequence which satisfy the above condition is always greater than $n-\frac{n}{2}$ or $n-\frac{n-1}{2}$ for the case $n$ is even or odd, respectively. Therefore

$$
\delta\left(\left\{k \leq n:\left|x_{k}\right|>r=\frac{b t}{1-b}\right\}\right)>\lim _{n \rightarrow \infty} \frac{1}{n} \times \frac{n}{2}=\frac{1}{2} \text { or } \lim _{n \rightarrow \infty} \frac{1}{n} \times \frac{n+1}{2}=\frac{1}{2} .
$$

Thus

$$
\delta\left(\left\{k \leq n:\left|x_{k}\right|>r=\frac{b t}{1-b}\right\}\right) \neq 0 \text { for all } b \in(0,1) .
$$

Hence

$$
B_{x}^{(\mu, v)}=(0,1),
$$

and

$$
s t_{(\mu, v)}-\lim \sup x=1 .
$$

The above sequence has two subsequences

$$
x=\left(x_{n_{i}}\right) \text { where } x_{n_{i}}=1 \text { for each } n_{i} \in\{3,5,7,11,13, \ldots\},
$$


and

$$
x=\left(x_{n_{j}}\right) \text { where } x_{n_{j}}=0 \text { for each } n_{j} \in\{2,6,8,10,12, \ldots\},
$$

$i, j \in \mathbb{N}$; which are of positive density and clearly convergent to 1 and 0 , respectively. Therefore, $x$ is not statistically convergent. Similarly, we have

$$
A_{x}^{(\mu, v)}=(0,1),
$$

and

$$
s t_{(\mu, v)}-\lim \inf x=0 .
$$

Hence the set of statistical cluster points of $x$ is $\{0,1\}$, where $s t_{(\mu, v)}$ - lim inf $x=$ least element and $s t_{(\mu, v)}$ - lim sup $x=$ greatest element of the above set.

This observation suggests the main idea of our first theorem of the following section.

\section{Main results}

The following results are analogs of the results due to Fridy and Orhan [6], while the proofs are different which show the technique to work with IFN-spaces. We observe that in contrast to the real case here from the definition limit sup cannot be infinite, as it can be at most 1 .

Theorem 3.1. Let $b=s t_{(\mu, v)}-\lim \sup x$. Then for every positive numbers $t$ and $\gamma$

$$
\left.\begin{array}{l}
\delta\left(\left\{k: \mu\left(x_{k} ; t\right)<1-b+\gamma \text { or } v\left(x_{k} ; t\right)>b-\gamma\right\}\right) \neq 0, \text { and } \\
\delta\left(\left\{k: \mu\left(x_{k} ; t\right)<1-b+\gamma \text { or } v\left(x_{k} ; t\right)>b+\gamma\right\}\right) \neq 0 .
\end{array}\right\}
$$

Conversely, if (1) holds for every positive $t$ and $\gamma$ then $b=s t_{(\mu, v)}-\lim \sup x$.

Proof. Let $b=s t_{(\mu, v)}$-lim sup $x$, where $b$ be finite. Then

$$
\delta\left(\left\{k: \mu\left(x_{k} ; t\right)<1-b \text { or } v\left(x_{k} ; t\right)>b\right\}\right) \neq 0 .
$$

Since $\mu\left(x_{k} ; t\right)<1-b+\gamma$ or $v\left(x_{k} ; t\right)>b-\gamma$ for every $k$ and for any $t, \gamma>0$,

$$
\delta\left(\left\{k \leq n: \mu\left(x_{k} ; t\right)<1-b+\gamma \text { or } \nu\left(x_{k} ; t\right)>b-\gamma\right\}\right) \neq 0 .
$$

Now applying the definition of $s t_{(\mu, v)}$ - $\lim \sup x$ we have $1-b$ as the least value and $b$ as the greatest value satisfying (2).

Now if possible,

$$
\mu\left(x_{k} ; t\right)<1-b-\gamma \text { or } \nu\left(x_{k} ; t\right)>b+\gamma \text { for some } \gamma>0 .
$$

Then $1-b-\gamma$ and $b+\gamma$ are another values with $1-b-\gamma<1-b$ and $b+\gamma>b$ which satisfies (2). This observation contradicts the fact that $1-b$ and $b$ are least and greatest values, respectively, which satisfies the above condition.

Hence,

$$
\delta\left(\left\{k \leq n: \mu\left(x_{k} ; t\right)<1-b-\gamma \text { or } v\left(x_{k} ; t\right)>b+\gamma\right\}\right)=0 \text { for every } \gamma>0 .
$$

Conversely, if (1) holds for every positive $t$ and $\gamma$, then

$$
\delta\left(\left\{k \leq n: \mu\left(x_{k} ; t\right)<1-b+\gamma \text { or } \nu\left(x_{k} ; t\right)>b-\gamma\right\}\right) \neq 0 .
$$


and

$$
\delta\left(\left\{k \leq n: \mu\left(x_{k} ; t\right)<1-b-\gamma \text { or } v\left(x_{k} ; t\right)>b+\gamma\right\}\right)=0 .
$$

Therefore

$$
\delta\left(\left\{k \leq n: \mu\left(x_{k} ; t\right) \leq 1-b \text { or } v\left(x_{k} ; t\right) \geq b\right\}\right) \neq 0
$$

and

$$
\delta\left(\left\{k \leq n: \mu\left(x_{k} ; t\right)=1-b \text { or } v\left(x_{k} ; t\right)=b\right\}\right)=0 .
$$

That is

$$
\delta\left(\left\{k \leq n: \mu\left(x_{k} ; t\right)<1-b \text { or } v\left(x_{k} ; t\right)>b\right\}\right) \neq 0 \text { for every } t>0 .
$$

Hence $b=s t_{(\mu v)}-\lim \sup x$.

This completes the proof of the theorem.

The dual statement for $s t_{(\mu, v)}-\lim$ inf $x$ can also be proved similarly.

Theorem 3.1'. Let $a=s t_{(\mu, v)}-\lim \inf x$. Then for every positive number $t$ and $\gamma$

$$
\left.\begin{array}{l}
\delta\left(\left\{k \leq n: \mu\left(x_{k} ; t\right)>1-a-\gamma \text { or } \nu\left(x_{k} ; t\right)<a+\gamma\right\}\right) \neq 0, \text { and } \\
\delta\left(k \leq n: \mu\left(x_{k} ; t\right)>1-a+\gamma \text { or } \nu\left(x_{k} ; t\right)<a-\gamma\right)=0 .
\end{array}\right\}
$$

Conversely, if (1') holds for every positive $t$ and $\gamma$ then $a=s t_{(\mu, v)}-\lim \inf x$.

Remark. From the definition of statistical cluster points we see that Theorems 3.1 and 3.1' can be interpreted as saying that $s t_{(\mu \nu)}$ - $\lim \sup x$ and $s t_{(\mu, v)}-\lim \inf x$ are the greatest and the least statistical cluster points of $x$, respectively.

Theorem 3.2. For any sequence $x, s t_{(\mu, v)}-\lim \inf x \leq s t_{(\mu, v)}-\lim \sup x$.

Proof. First consider the case in which $s t_{(\mu, v)}-\lim \sup x=0$, which implies that

$$
B_{x}^{(\mu, \nu)}=\emptyset
$$

Then for every $b \in(0,1)$,

$$
B_{x}^{(\mu, v)}=\delta\left(\left\{k: \mu\left(x_{k} ; t\right)<1-b \text { or } v\left(x_{k} ; t\right)>b\right\}\right)=0,
$$

that is

$$
\delta\left(\left\{k: \mu\left(x_{k} ; t\right) \geq 1-b \text { or } v\left(x_{k} ; t\right) \leq b\right\}\right)=1 .
$$

Also for every $a \in(0,1)$, we have

$$
\delta\left(\left\{k: \mu\left(x_{k} ; t\right)>1-a \text { or } v\left(x_{k} ; t\right)<a\right\}\right) \neq 0 .
$$

Hence, $s t_{(\mu, v)}-\lim \inf x=0$.

The case in which $s t_{(\mu, v)}-\lim \sup x=1$, is trivial.

Suppose that $b=s t_{(\mu, v)}-\lim \sup x$, and $a=s t_{(\mu, v)}$ - $\lim \inf x$; where $a$ and $b$ are finite.

Now for given any $\gamma$, we show that $1-b-\gamma \in A_{x}^{(\mu, v)}$. 
By Theorem 3.1,

$$
\delta\left(\left\{k: \mu\left(x_{k} ; t\right)<1-b-\frac{\gamma}{2} \text { or } v\left(x_{k} ; t\right)>b+\frac{\gamma}{2}\right\}\right)=0, \quad \text { where } 1-b=\text { least upper bound of } B_{x}^{(\mu, v)} .
$$

Therefore

$$
\delta\left(\left\{k: \mu\left(x_{k} ; t\right) \geq 1-b-\frac{\gamma}{2} \text { or } v\left(x_{k} ; t\right) \leq b+\frac{\gamma}{2}\right\}\right)=1,
$$

which in turn gives

$$
\delta\left(\left\{k: \mu\left(x_{k} ; t\right)>1-b-\gamma \text { or } v\left(x_{k} ; t\right)<b+\gamma\right\}\right)=1 .
$$

Hence, $1-b-\gamma \in A_{x}^{(\mu, v)}$.

By definition

$$
a=\inf A_{x}^{(\mu, \nu)},
$$

so we conclude that

$$
1-b-\gamma \leq 1-a,
$$

and since $\gamma$ is arbitrary,

$$
1-b \leq 1-a,
$$

that is

$$
\begin{aligned}
& -b \leq-a, \\
& a \leq b .
\end{aligned}
$$

This completes the proof of the theorem.

Theorem 3.3. In an intuitionistic fuzzy normed space $(X, \mu, v, *, \diamond)$, the statistically bounded sequence $x$ is statistically convergent if and only if

$$
s t_{(\mu, v)}-\lim \inf x=s t_{(\mu, v)}-\lim \sup x .
$$

Proof. Let $\alpha, \beta$ be $s t_{(\mu, v)}$ - lim inf $x$ and $s t_{(\mu, v)}$ - lim sup $x$, respectively. Now we assume that $s t_{(\mu v)^{-}} \lim x=L$. Then for every $\epsilon>0$ and $b \in(0,1)$,

$$
\delta\left(\left\{k: \mu\left(x_{k} ; t\right) \leq 1-b \text { or } v\left(x_{k} ; t\right) \geq b\right\}\right)=0,
$$

so that

$$
\delta\left(\left\{k: \mu\left(x_{k} ; \frac{t}{2}\right) * \mu\left(L ; \frac{t}{2}\right) \leq 1-b \text { or } v\left(x_{k} ; \frac{t}{2}\right) \diamond v\left(L ; \frac{t}{2}\right) \geq b\right\}\right)=0 .
$$

Let for every $t>0$,

$$
\sup _{t} \mu\left(x_{k} ; \frac{t}{2}\right)=1-b_{1} \text { and } \sup _{t} \mu\left(L ; \frac{t}{2}\right)=1-b_{2}
$$

or

$$
\inf _{t} v\left(x_{k} ; \frac{t}{2}\right)=b_{1} \text { and } \inf _{t} v\left(L ; \frac{t}{2}\right)=b_{2}
$$


such that

$$
\left(1-b_{1}\right) *\left(1-b_{2}\right) \leq 1-b \text { or } b_{1} \diamond b_{2} \geq b .
$$

Then

$$
\delta\left(\left\{k: \mu\left(x_{k} ; \frac{t}{2}\right) \leq 1-b_{1} \text { or } v\left(x_{k} ; \frac{t}{2}\right) \geq b_{1}\right\}\right)=0,
$$

and therefore

$$
\delta\left(\left\{k: \mu\left(x_{k} ; \frac{t}{2}\right)<1-b_{1}-\gamma \text { or } v\left(x_{k} ; \frac{t}{2}\right)>b_{1}+\gamma\right\}\right)=0 \text { for every } \gamma>0 .
$$

Now applying Theorem 3.1 and the definition of $s t_{(\mu v)^{-}} \lim \sup x$, we get

$$
\delta\left(\left\{k: \mu\left(x_{k} ; \frac{t}{2}\right)<1-\beta-\gamma \text { or } v\left(x_{k} ; \frac{t}{2}\right)>\beta+\gamma\right\}\right)=0 \text { for every } \gamma>0 .
$$

From (3.3) and (3.4) and by the definition of $s t_{(\mu \nu v)}-\lim \sup x$, we get

$$
1-b_{1}-\gamma \leq 1-\beta-\gamma \text { or } b_{1}+\gamma \geq \beta+\gamma,
$$

that is,

$$
\beta \leq b_{1} \text {. }
$$

Now we find those $k$ such that

$$
\mu\left(x_{k} ; \frac{t}{2}\right)>1-b_{1}+\gamma \text { or } v\left(x_{k} ; \frac{t}{2}\right)<b_{1}-\gamma .
$$

We can easily observe that no such $k$ exists which satisfy (3.1) and above condition together.

Therefore this implies that

$$
\delta\left(\left\{k: \mu\left(x_{k} ; \frac{t}{2}\right)>1-b_{1}+\gamma \text { or } v\left(x_{k} ; \frac{t}{2}\right)<b_{1}-\gamma\right\}\right)=0 .
$$

Since $\alpha=s t_{(\mu, v)}-\lim \inf x$, by Theorem 3.1', we get

$$
\delta\left(\left\{k: \mu\left(x_{k} ; \frac{t}{2}\right)>1-\alpha+\gamma \text { or } v\left(x_{k} ; \frac{t}{2}\right)<\alpha-\gamma\right\}\right)=0 .
$$

By the definition of $s t_{(\mu, v)}-\lim \inf x$, we have

$$
1-\alpha+\gamma \leq 1-b_{1}+\gamma \text { or } \alpha-\gamma \geq b_{1}-\gamma,
$$

that is,

$$
b_{1} \leq \alpha .
$$

From (3.4) and(3.5), we get $\beta \leq \alpha$. Now combining Theorem 3.2 and the above inequality, we conclude $\alpha=\beta$.

Conversely, suppose that $\alpha=\beta$ and let $\sup _{t} \mu(L, t)=1-\alpha \operatorname{or}_{\inf } v(L, t)=\alpha$. Then for any $\gamma>0$, Theorems 3.1 and 3.1' will together imply that 


$$
\delta\left(\left\{k: \mu\left(x_{k} ; \frac{t}{2}\right)<1-\alpha+\frac{\gamma}{2} \text { or } \nu\left(x_{k} ; \frac{t}{2}\right)>\alpha-\frac{\gamma}{2}\right\}\right)=0,
$$

and

$$
\delta\left(\left\{k: \mu\left(x_{k} ; \frac{t}{2}\right)>1-\alpha+\frac{\gamma}{2} \text { or } \nu\left(x_{k} ; \frac{t}{2}\right)<\alpha-\frac{\gamma}{2}\right\}\right)=0 .
$$

Now

$$
1-\alpha \geq \mu(L ; t)=\mu\left(x_{k}-\left(x_{k}-L\right) ; t\right) \geq \mu\left(x_{k} ; \frac{t}{2}\right) * \mu\left(x_{k}-L ; \frac{t}{2}\right),
$$

and

$$
\alpha \leq v(L ; t)=v\left(x_{k}-\left(x_{k}-L\right) ; t\right) \leq v\left(x_{k} ; \frac{t}{2}\right) \diamond v\left(x_{k}-L ; \frac{t}{2}\right) .
$$

Therefore

$$
\mu\left(x_{k} ; \frac{t}{2}\right) * \mu\left(x_{k}-L ; \frac{t}{2}\right) \leq 1-\alpha \text { or } v\left(x_{k} ; \frac{t}{2}\right) \diamond v\left(x_{k}-L ; \frac{t}{2}\right) \geq \alpha
$$

Let $\sup _{t}\left\{\mu\left(x_{k}-L ; \frac{t}{2}\right)\right\}=1-a_{1}$ or $\inf _{t}\left\{v\left(x_{k}-L ; \frac{t}{2}\right)\right\}=a_{1}$, where $a_{1} \in(0,1)$ and (3.7) and (3.9) hold. Then

$$
\delta\left(\left\{k: \mu\left(x_{k}-L ; \frac{t}{2}\right)<1-\alpha_{1}-\frac{\gamma}{2} \text { or } \nu\left(x_{k}-L ; \frac{t}{2}\right)>\alpha_{1}+\frac{\gamma}{2}\right\}\right)=0,
$$

which is true for all $\gamma>0$. Hence

$$
\delta\left(\left\{k: \mu\left(x_{k}-L ; \frac{t}{2}\right) \leq 1-\alpha_{1} \text { or } v\left(x_{k}-L ; \frac{t}{2}\right) \geq \alpha_{1}\right\}\right)=0
$$

which is true for all $a \leq a_{1} \in(0,1)$, because $1-a_{1}$ is the least upper bound or $a_{1}$ is the greatest lower bound.

Now repeat the process by taking (3.8) and (3.9) instead of (3.7) and (3.9). If (3.8) and (3.9) are satisfied, then $\inf _{t} \mu\left(x_{k}-L ; \frac{t}{2}\right)=1-a_{1}$ or $\sup _{t} v\left(x_{k}-L ; \frac{t}{2}\right)=a_{1}$,

On contrary suppose that $1-a_{1} \neq \inf _{t} \mu\left(x_{k}-L ; \frac{t}{2}\right)$ or $a_{1} \neq \sup _{t} \nu\left(x_{k}-L ; \frac{t}{2}\right)$ and conditions (3.8) and (3.9) be satisfied. This implies that there exists some $r \in(0,1)$ such that either $1-r=\mu\left(x_{k}-L ; \frac{t}{2}\right)$ or $r=v\left(x_{k}-L ; \frac{t}{2}\right)$ for some $t>0$ where $1-a_{1}$ $>1-r$ or $a_{1}<r$.

As (3.8) and (3.9) are satisfied, and let us suppose that $\inf _{t} \mu\left(x_{k}-L ; \frac{t}{2}\right)=1-a_{2}$ or $\sup _{t} v\left(x_{k}-L ; \frac{t}{2}\right)=a_{2}$.

Then

$$
1-a_{1}>1-a_{2} \text { or } a_{1}<a_{2}
$$

and from (3.9), we get

$$
\mu\left(x_{k}-L ; \frac{t}{2}\right) *\left(1-a_{2}\right) \leq 1-\alpha \text { or } v\left(x_{k}-L ; \frac{t}{2}\right) \diamond \alpha_{2} \geq \alpha
$$


Using (3.8), we get

$$
\left(1-\alpha+\frac{\gamma}{2}\right) *\left(1-a_{2}\right) \leq 1-\alpha \text { or } v\left(\alpha-\frac{\gamma}{2}\right) \diamond\left(\alpha_{2}\right) \geq \alpha \text { for all } \gamma>0 .
$$

Clearly,

$$
\left(1-\alpha+\frac{\gamma}{2}\right) *\left(1-a_{2}\right) \leq 1-\alpha \text { or }\left(\alpha+\frac{\gamma}{2}\right) \diamond\left(\alpha_{2}\right) \geq \alpha \text { for all } \gamma>0 .
$$

Now

$$
1-a_{1}=\sup _{t} \mu\left(x_{k}-L ; \frac{t}{2}\right) \text { or } \alpha_{1}=\inf _{t} v\left(x_{k}-L ; \frac{t}{2}\right)
$$

where $a_{1} \in(0,1)$ and which satisfy (3.7) and (3.9).

From (3.11) we conclude that $1-a_{2}$ is another value satisfying (3.7) and (3.9).

Hence

$$
1-a_{1}<1-a_{2} \text { or } a_{2}<a_{1} .
$$

This contradicts (3.10). Hence $1-a_{1}=\inf _{t} \mu\left(x_{k}-L ; \frac{t}{2}\right)$ or $a_{1}=\sup _{t} v\left(x_{k}-L ; \frac{t}{2}\right)$ satisfying conditions (3.8) and (3.9).

Therefore the inequality becomes true for all $a \geq a_{1} \in(0,1)$, because $1-a_{1}$ is the greatest lower bound, and hence

$$
\delta\left(\left\{k: \mu\left(x_{k}-L ; \frac{t}{2}\right) \leq 1-\alpha \text { or } v\left(x_{k}-L ; \frac{t}{2}\right) \leq \alpha\right\}\right)=0,
$$

for each $t>0$ and $a \in(0,1)$. Therefore

$$
s t_{(\mu, v)}-\lim x=L .
$$

This completes the proof of the theorem.

\section{Acknowledgements}

The authors would like to thank the Research Deanship at King Abdulaziz University for its financial support under grant number 147/130/1431.

\section{Author details}

${ }^{1}$ Department of Mathematics, King Abdulaziz University, Jeddah 21589, Saudi Arabia ${ }^{2}$ Department of Computer Science, South Asian University, New Delhi 110070, India ${ }^{3}$ Department of Mathematics, Aligarh Muslim University, Aligarh 202002, India

\section{Authors' contributions}

The present study was proposed by MAA and AA. Definitions 2.1-2.4 and Theorem 3.1, 3.2 and 3.3 were given by MAA, AA and QMDL. MM gave the final shape to the present work by incorporating some necessary suggestions. All authors read and approved the final manuscript.

\section{Competing interests}

The authors declare that they have no competing interests.

Received: 22 December 2011 Accepted: 23 April 2012 Published: 23 April 2012

\section{References}

1. Fast, H: Sur la convergence statistique. Colloq Math. 2, 241-244 (1951)

2. Mursaleen, M, Cakan, C, Mohiuddine, SA, Savas, E: Generalized statistical convergence and statistical core of double sequences. Acta Math Sinica. 26(11):2131-2144 (2010)

3. Mursaleen, M, Edely, OHH: Statistical convergence of double sequences. J Math Anal Appl. 288, 223-231 (2003)

4. Fridy, JA: On statistical convergence. Analysis. 5, 301-313 (1985)

5. Fridy, JA: Statistical limit points. Proc Am Math Soc. 118, 1187-1192 (1993) 
6. Fridy, JA, Orhan, C: Statistical limit superior and limit inferior. Proc Am Math Soc. 125, 3625-3631 (1997)

7. Aytar, S: Statistical limit points of sequences of fuzzy numbers. Inf Sci. 165, 129-138 (2004)

8. Aytar, S, Pehlivan, S: Statistical cluster and extreme limit points of sequences of fuzzy numbers. Inf Sci. 177, 3290-3296 (2007)

9. Savaş, E, Mursaleen, M: On statistically convergent double sequences of fuzzy numbers. Inf Sci. 162, 183-192 (2004)

10. Karakus, S, Demirci, K, Duman, O: Statistical convergence on intuitionistic fuzzy normed spaces. Chaos Solitons \& Fractals. 35, 763-769 (2008)

11. Mursaleen, M, Mohiuddine, SA: On lacunary statistical convergence with respect to the intuitionistic fuzzy normed space. J Comput Appl Math. 233(2):142-149 (2009)

12. Mursaleen, M, Mohiuddine, SA: Statistical convergence of double sequences in intu-itionistic fuzzy normed spaces. Chaos Solitons \& Fractals. 41, 2414-2421 (2009)

13. Mursaleen, M, Mohiuddine, SA: On the ideal convergence of double sequences in intu-tionistic fuzzy normed spaces. Comput Math Appl. 59, 603-611 (2010)

14. Saadati, R, Park, JH: On the intuitionistic fuzzy topological spaces. Chaos Solitons \& Fractals. 27, 331-344 (2006)

15. Mursaleen, M, Lohani, QMD: Intuitionistic fuzzy 2-normed space and some related concepts. Chaos Solitons \& Fractals. 42, 224-234 (2009)

16. Mursaleen, M, Lohani, QMD, Mohiuddine, SA: Intuitionistic fuzzy 2-metric space and its completion. Chaos Solitons \& Fractals. 42, 1258-1265 (2009)

17. Freedman, AR, Sember, JJ, Raphael, M: Some Cesàro type summability spaces. Proc Lond Math Soc. 37, 508-520 (1978)

18. Šalát, T: On statistically convergent sequences of real numbers. Math Slovaca. 30, 139-150 (1980)

doi:10.1186/1029-242X-2012-96

Cite this article as: Alghamdi et al: Statistical limit superior and limit inferior in intuitionistic fuzzy normed

spaces. Journal of Inequalities and Applications 2012 2012:96.

\section{Submit your manuscript to a SpringerOpen ${ }^{\circ}$} journal and benefit from:

Convenient online submission

- Rigorous peer review

- Immediate publication on acceptance

- Open access: articles freely available online

- High visibility within the field

- Retaining the copyright to your article

Submit your next manuscript at $\gg$ springeropen.com 